\title{
Problems posed by potential gonococcal vaccines viewed from the vantage point of a control agency
}

\author{
JOHN B. ROBBINS \\ From the Division of Bacterial Products Bureau of Biologics, Bethesda, Maryland
}

SUMMARY Progress in the characterisation of Neisseria gonorrhoeae and other bacterial pathogens has suggested that immunoprophylaxis for gonorrhoea may be possible despite the well-known propensity for reinfection. Pili, outer membrane proteins, a capsular polysaccharide, and the lipopolysaccharide may be important gonococcal virulence factors, and immune components (probably antibodies) to more than one of these antigens may be required to confer immunity. A study of antigenic polymorphism of these structures should identify disease isolates more precisely and provide information about the relationship between variants of these gonococcal structures and gonococcal virulence.

\section{Introduction}

Recently, the potential for immunoprophylaxis against Neisseria gonorrhoeae (gonococci) has received attention from research laboratories, despite the well-known observation that disease caused by this organism does not confer immunity against reinfection (Tramont et al., 1976). Progress in the field of gonococcal-host interaction has been enriched by several observations:

1. The demonstration that pili or fimbriae were the structures related to the morphology of colony variants that characterise disease isolates (Kellogg et al., 1963, 1968). It has been suggested that these pili are required for adherence of gonococci to genitourinary mucous membranes (Swanson et al., 1971). Some investigators have proposed an additional pathogenic role for pili as anti-phagocytic structures. In these studies, serum antipilar antibody inhibited adherence and facilitated opsonisation of the pilated gonococci (Punsalang and Sawyer, 1973). Antigenic polymorphism without demonstrable cross-reactivity of pili has been described (Buchanan, 1975; Novotny and Turner, 1975).

\footnotetext{
Address for reprints: Dr J. B. Robbins, Division of Bacterial Products Bureau of Biologics, Bethesda, Maryland 20014, USA

Received for publication 15 December 1976
}

2. Antigenic polymorphism of gonococcal outer membrane proteins and lipopolysaccharides has been reported (Apicella, 1974; Johnston and Gotschlich, 1974; Johnston et al., 1976). In addition to their potential as protective immiunogens, these structural characteristics permit more precise identification of disease isolates.

3. Unique biochemical characteristics of gonococci have been isolated from blood-borne infections, such as septicaemia and arthritis (Knapp and Holmes, 1975).

4. A morphological characteristic of pathogenic gonococci grown on trypsin-free medium is that a halo-like effect is achieved if individual colonies are viewed using indirect light (Swanson, 1977).

5. There is morphological evidence of a capsular polysaccharide from fresh isolates. This capsular polysaccharide is extremely susceptible to mechanical agitation and is quickly lost during passage on conventional medium (Richardson and Sadoff, 1977; Swanson, personal communication).

These discoveries of gonococcal physiology and structure, together with observations on bacterial components used for vaccines of Vibrio cholerae and Group B Neisseria meningitidis, have awakened optimism that immunoprophylaxis against gonorrhoea may be possible. 
Concepts to be considered in the development of a gonococcal vaccine

Prevention of a bacterial disease by immunological mechanisms largely confined to the mucosal surface poses several problems. In this regard, the pathogenic mechanisms of $V$. cholerae may be considered as a model for development of gonococcal vaccineinduced immunity. Data derived from animal models and electron micrographs have indicated that at least three components of $V$. cholerae are critical pathogenic structures and may be considered as potential immunogens. The first component is extracellular toxin, and the second the somatic antigen. Recent studies by Holmgren and Svennerholm (1977) have shown that the protective effect of serum antibodies to both the somatic antigen and toxin exceeds the sum of antibody-mediated effect to these components individually (Holmgren and Svennerholm, 1977), thus, the somatic antigen, toxin, and adherent factor(s). The third component is suggested by in vivo studies from Finkelstein's laboratory. Using morphological criteria, it has been shown that $V$. cholerae adheres in a specific fashion to the intestinal mucosa. Curiously, the adherent organisms had few pili as conventionally studied (Nelson et al., 1976). This 'adhesive' structure(s) (pili?) of $V$. cholerae may be an important effector immunogen for eliciting protective immunity by vaccines for cholera, a non-invasive organism which elicits a mucosal disease. These experiments also indicate that parenteral immunisation to elicit serum antibodies may be an effective approach in preventing disease and may be accomplished without applying the immunogen directly to the mucosal surfaces.

A similar synergism between serum antibodies to both the capsular polysaccharide and outer membrane proteins (serotype antigens) for inducing protection against Group B $N$. meningitidis in the chick embryo model was reported (Frasch et al., 1976). The necessity for inducing immunity to a multiplicity of bacterial components may also be considered in immunoprophylaxis experiments against gonorrhoea. Thus, if the pilated-mediated attachment, outer cell wall membrane proteins, and endotoxin are all necessary for the pathogenesis of gonorrhoea, immune components may have to be induced to each for effective immunity.

Antigenic polymorphorism-implication for vaccines and surveillance

Another important consideration is the possibility that there is a highly diverse system of protective antigens. If there is no or little cross-reactivity among the many antigenic variants of gonococcal pili, lipolysaccharide, and outer membrane proteins, possibly immunity may have to be induced to each component of these bacterial structures. Furthermore, as in the cases of many diverse structures such as Escherichia coli, capsular polysaccharides, and somatic antigens, there may be no relationship between these functionally and morphologically different antigenic variants of these structures and disease isolates (Sarff et al., 1975). For example, the most difficult possibility is that the biosynthesis of these three proposed protective antigens is unrelated; if each system has at least 10 antigenic variants, the gonococcal vaccines will indeed be complex. This may not be the case, as only three of the 13 antigenically unique outer membrane proteins of Group B $N$. meningitidis are associated with organisms causing disease (Frasch and Chapman, 1972). Surveillance studies of the antigenic profile of disease isolates will be necessary to decide among these several possibilities.

Is an animal model necessary for effective vaccine control?

The example of meningococcal polysaccharide vaccine should serve to indicate that adequate physical-chemical characterisation of protective antigens is sufficient to ensure their effectiveness as vaccines (World Health Organisation, 1976). It is unlikely that an effective animal model for gonorrhoea will be established, considering the nature of the transmission of the disease and the difficulty in obtaining and maintaining primates (Arko et al., 1974). However, the mechanism of vaccine-induced immunity can be related to the pathogenesis of disease-that is, antibodies to pili can inhibit gonococcal attachment in vitro, antibodies to outer membrane proteins may facilitate opsonisation, etc., and it may be possible to prepare standard gonococcal components for vaccines by physicochemical and immunochemical methods without the need to establish potency in an animal model. Not all human diseases occur or may be induced in animals. It is well to consider the long experience with typhoid vaccines, shown to be protective in a contrived animal model, but whose efficacy in the field leaves much to be desired. Conversely, consider the problem of potency testing of diptheria and tetanus toxoids in laboratory animals, which, despite long experience of many laboratories, still cannot reliably be assayed in an animal model to predict accurately their effectiveness in inducing a primary serum antibody response in human infants. Thus, techniques for accurate and reliable identification of the physical-chemical characteristics related to immunogenicity must be developed for 
these gonococcal structures as their pathogenic role is uncovered. A corollary of this hypothesis is that efforts should be directed towards achieving the highest purity for each potential bacterial component by standardised methods of production and analysis.

\section{Does the lack of disease-induced immunity obviate the need for vaccine development?}

The lack of disease-induced immunity may not be due to the extreme antigenic diversity of gonococci that seems to emerge from recent studies. Another possibility is that infection on the genitourinary surfaces does not generate sufficient immunogenic stimulus for disease protection. Tetanus, pertussis, and smallpox are examples for which effective vaccines have been developed, despite the fact that disease with these organisms does not reliably confer long-lived immunity. It should not deter us in our quest for vaccines against gonorrhoea whether or not disease can confer immunity. It may be that vaccination may do what the disease process with the organisms cannot.

\section{References}

Apicella, M. (1974). Antigenically distinct populations of Neisseria gonorrhoeae. Isolation and characterization of the responsible determinants. Journal of Infectious Diseases, 130, 619-626.

Arko, R. M., Kraus, S. J., Brown, W. J., Buchanan, T. M., and Kuhn, U. S. G. (1974). Neisseria gonorrhoeae: effects of systemic immunization on resistance of chimpanzees to urethral infection. Journal of Infectious Diseases, 130, 160-168.

Buchanan, T. M. (1975). Antigenic heterogeneity of gonococcal pili. Journal of Experimental Medicine, 141, 1470-1475.

Frasch, C. E., and Chapman, S. (1972). Classification of Neisseria meningitidis group B into distinct serotypes. III. Application of a new bactericidal inhibition technique to the distribution of serotypes among cases and carriers. Journal of Infectious Diseases, 127, 149-155.
Frasch, C. E., Parkes, L., McNeilis, R. M., and Gotschlich, E. C. (1976). Protection against group B meningococcal disease. I. Comparison of group-specific and type-specific protection in the chick embryo model. Journal of Experimental Medicine, 144, 319329.

Holmgren, J., and Svennerholm, A. M. (1977). Mechanisms of disease and immunity in cholera. In Symposium on Current Status and Prospects for Improved and New Bacterial Vaccines. Journal of Infectious Diseases, 136, in press.

Johnston, K. H., and Gotschlich, E. C. (1974). Isolation and characterization of the outer membrane of Neisseria gonorrhoeae. Journal of Bacteriology, 119, 886-906.

Johnston, K. H., Holmes, K. K., and Gotschlich, E. C. (1976). The serological classification of Neisseria gonorrhoeae. I. The isolation of the outer membrane complex for serotype specificity. Journal of Experimental Medicine, 143, 907-918.

Kellogg, D. S., Jr, Cohen, I. R., Norins, L. C., Schroeter, A. L., and Reising, G. (1968). Neisseria gonorrhoeae. II. Colonial variation and pathogenicity during 35 months in vitro. Journal of Bacteriology, 96, 596-605.

Kellogg, D. S., Jr, Peacock, W. L., Deacon, W. E., Brown, L., and Pirkle, C. (1963). Neisseria gonorrhoeae. I. Virulence related to colonial variation. Journal of Bacteriology, 85, 1274-1290.

Knapp, J. S., and Holmes, K. K. (1975). Disseminated gonococcal infections caused by Neisseria gonorrhoeae with unique nutritional requirements. Journal of Infectious Diseases, 132, 204-208.

Nelson, E. T., Clements, J. D., and Finkelstein, R. A. (1976). Vibrio cholerae adherence and colonization in experimental cholera: electron microscopic studies. Infection and Immunity, 14, 527-547.

Novotny, P., and Turner, W. H. (1975). Immunological heterogeneity of pili of Neisseria gonorrhoeae. Journal of General Microbiology, 89, 87-92.

Punsalang, A. P., and Sawyer, W. D. (1973). Role of pili in the virulence of Neisseria gonorrhoeae. Infection and Immunity, 8, 255-263.

Richardson, W. P., and Sadoff, J. (1977). Production of a capsule by Neisseria gonorrhoeae. Infection and Immunity, 15, 663-664.

Sarff, L. D., McCracken, G. H., Schiffer, M. S., Glode, M. P., Robbins, J. B., Ørskov, I., and Ørskov, F. (1975). Epidemiology of Escherichia coli $\mathrm{K} 1$ in healthy and diseased newborns. Lancet, 1, 1099-1104.

Swanson, J. (1977). Studies on the gonococci. In Symposium on Current Status and Prospects for Improved and New Bacterial Vaccines. Journal of Infectious Diseases, 136, in press.

Swanson, J., Kraus, S. J., and Gotschlich, E. C. (1971). Studies on gonococcal infection. I. Pili and zones of adhesion: their relation to gonococcal growth patterns. Journal of Experimental Medicine, $134,250-257$.

Tramont, E. C., Griffiss, J. M., Rose, D., Brooks, G. F., and Artenstein, M. S. (1976). Clinical correlation of strain differentiation of Neisseria gonorrhoeae. Journal of Infectious Diseases. 134, 128-134.

World Health Organisation (1976). Technical report series 588: Cerebrospinal meningitis control: WHO Expert Committee on Biological Standardization. Technical report series 594: Requirements for meningococcal polysaccharide vaccine. WHO: Geneva. 